\title{
Temperature Measurement by a Nanoscale Electron Probe using Energy Gain and Loss Spectroscopy
}

Juan Carlos Idrobo ${ }^{1}$, Andrew R. Lupini ${ }^{2}$, Tianli Feng ${ }^{3}$, Raymond R. Unocic ${ }^{1}$, Franklin S. Walden ${ }^{4}$, Daniel S. Gardiner ${ }^{4}$, Tracy C. Lovejoy ${ }^{5}$, Niklas Dellby ${ }^{5}$, Sokrates T. Pantelides ${ }^{3}$, and Ondrej L. Krivanek $^{5}$

1. Center for Nanophase Materials Sciences, Oak Ridge National Laboratory, Oak Ridge, TN, USA

2. Materials Science and Technology Division, Oak Ridge National Laboratory, Oak Ridge, TN, USA

3. Department of Physics and Astronomy and Department of Electrical Engineering and Computer Science, Vanderbilt University, Nashville, TN, USA

4. Protochips Company, 3800 Gateway Centre Blvd, Suite 306, Morrisville, NC, USA

5. Nion Company, 11511 NE 118th St, Kirkland, WA, USA

Correct thermal management is essential for the microelectronics industry [1]. Accuracy requires the use of a microscopy technique that is able to map the local temperature of a material with high spatial resolution. Local temperature measurements with nanoscale spatial resolution have recently been achieved by tracking the energy shifts of bulk plasmons as a function of temperature in a scanning transmission electron microscope (STEM) [2]. Another approach to measure temperature is based on a concept that originated in the 1960s and consists of determining the ratio of energy gains to energy losses of a fast electron beam interacting with low energy excitation such as phonons in a material [3-5].

Here, we use the phonon approach to measure the local temperature of nanomaterials by electron energy-gain and energy loss spectroscopy in a monochromated aberration-corrected STEM [6]. The experiments were performed using ORNL's Nion HERMES ${ }^{\mathrm{TM}}$ operated at $60 \mathrm{kV}$. Nanoflakes of hexagonal boron nitride (h-BN) were deposited on a Protochips Fusion MEMS-based heating chip, where the temperature was varied from $\sim 320 \mathrm{~K}$ to $\sim 1600 \mathrm{~K}$. Figure 1a shows the energy gain and loss peaks of an optical phonon mode of the h-BN as a function of temperature, which were obtained in an aloof scattering geometry. Figure $1 \mathrm{~b}$ shows the temperature obtained using the principle of detailed balance [7]. The experiments in this study show that by detecting gain peaks, the local temperature of a material can be obtained directly, based purely on statistical principles, and indirectly (using empirical fits). Combined with theory, energy-gain spectroscopy also enables the study of anharmonic effects in materials by probing phonons in the electron microscope at different temperatures [8].

\section{References:}

[1] E Pop, et al., Proc. IEEE 94 (2006), p. 1587.

[2] M Mecklenburg, et al., Science 347 (2015), p.629.

[3] H Boersch, et al., Phys. Rev. Lett. 17 (1966), p. 379.

[4] A Howie, Adv. Inst. Phys. Conf. Ser. 161 (1999), p. 311.

[5] FJ García de Abajo and M. Kociak, New J. Phys. 10 (2008), p. 073035.

[6] JC Idrobo, et al., Phys. Rev. Lett. (2016), in press.

[7] GL Squires, Thermal Neutron Scattering, (3rd edn Cambridge University Press 2012)

[8] Research supported by ORNL's Center for Nanophase Materials Sciences, which is a U.S. Department of Energy Office of Science User Facility (J.C.I. and R.R.U.), and by the Materials Sciences and Engineering Division Office of Basic Energy Sciences, U.S. Department of Energy (A.R.L.). This 
research used resources of the National Energy Research Scientific Computing Center, which is supported by the Office of Science of the U.S. Department of Energy under Contract No. DE-AC0205CH11231, and instrumentation within ORNL's Materials Characterization Core provided by UTBattelle, LLC under Contract No. DE-AC05-00OR22725 with the U.S. Department of Energy. This work used the Extreme Science and Engineering Discovery Environment (XSEDE). Theoretical work at Vanderbilt University was supported by DOE Award No. DE-FG02-09ER46554 and by the McMinn Endowment (T.L.F. and S.T.P.).
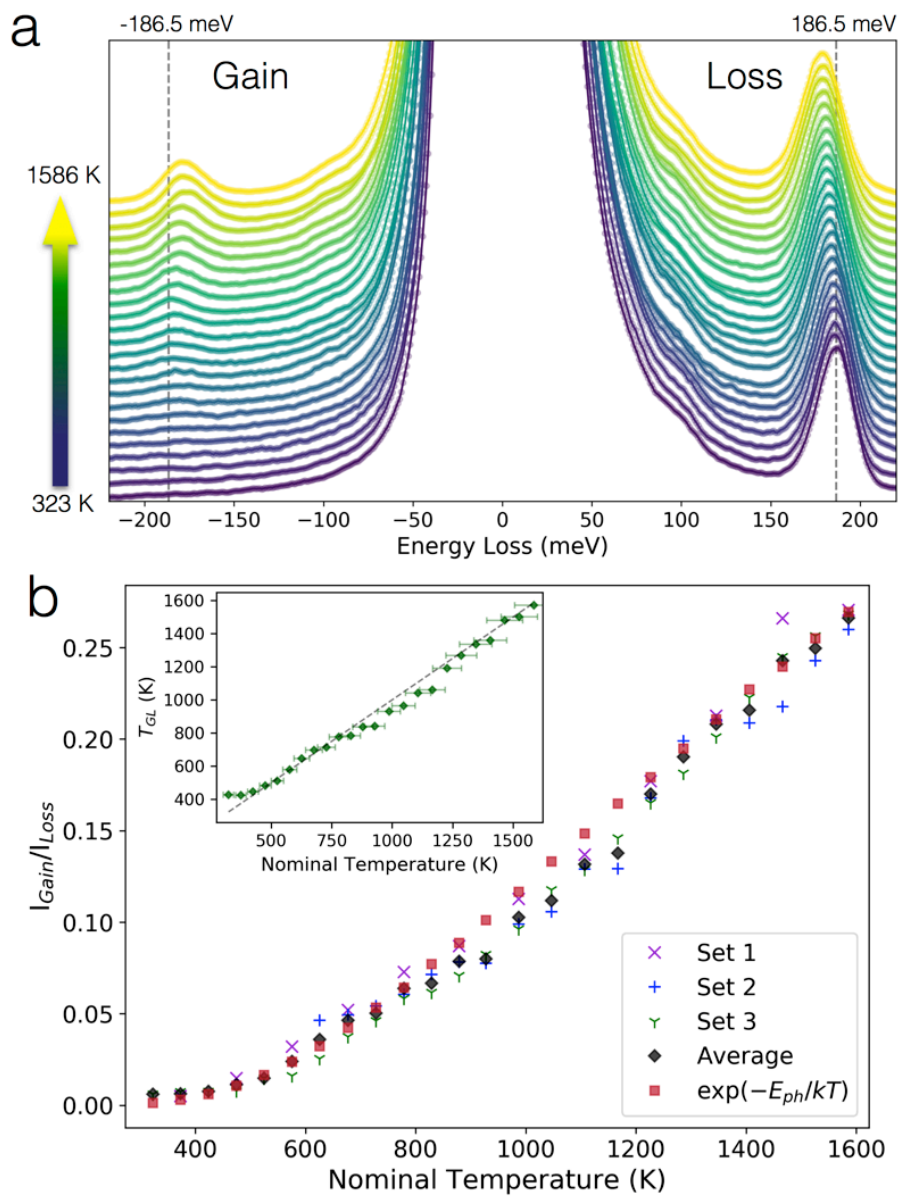

Figure 1. Electron energy-loss and energy gain spectra of h-BN as a function of temperature (a) Spectra acquired as function of temperature from $323 \mathrm{~K}$ to $1586 \mathrm{~K}$, with an average increment of $55 \mathrm{~K}$. (b) Gain and loss phonon peak intensity $\left(\mathrm{I}_{\text {Gain }} / \mathrm{I}_{\text {Loss }}\right)$ ratios of three different sets of measurements performed at different times (and the respective average). Inset in (b) shows the temperatures obtained by the principle of detailed balance $\mathrm{T}_{\mathrm{GL}}$ against the nominal temperature. Figure adapted from Ref. 6. 